\title{
Comprehensive analysis of in-hospital delirium after major surgical oncology procedures
}

Marco Bandini ${ }^{1,2,3^{*}}$; Michele Marchioni ${ }^{1,2,4^{*}}$; Felix Preisser ${ }^{1,2,5}$; Sebastiano Nazzani ${ }^{1,2,6}$; Zhe Tian $^{1,2}$; Markus Graefen ${ }^{5}$; Francesco Montorsi ${ }^{3}$; Fred Saad ${ }^{1,2}$; Shahrokh F. Shariat ${ }^{7}$; Luigi Schips $^{4}$; Alberto Briganti ${ }^{3}$; Pierre I. Karakiewicz ${ }^{1,2}$

${ }^{1}$ Cancer Prognostics and Health Outcomes Unit, University of Montreal Health Center (CHUM), Montreal, QC, Canada; ${ }^{2}$ Centre de recherche du Centre Hospitalier de l'Université de Montréal (CR-CHUM) and Division of Urology, CHUM, Montreal, QC, Canada; ${ }^{3}$ Division of Oncology/Unit of Urology, URI, IRCCS Ospedale San Raffaele, and Vita-Salute San Raffaele University, Milan, Italy; ${ }^{4}$ Department of Urology, SS Annunziata Hospital, "G. D'Annunzio" University of Chieti, Chieti, Italy; ${ }^{5}$ Martini Klinik, University Medical Center Hamburg-Eppendorf, Hamburg, Germany; ${ }^{6}$ Academic Department of Urology, IRCCS Policlinico San Donato, University of Milan, Milan, Italy; ${ }^{7}$ Department of Urology, Medical University of Vienna, Vienna, Austria ${ }^{*}$ Equal contributors

Cite as: Can Urol Assoc J 2019 September 27; Epub ahead of print. http://dx.doi.org/10.5489/cuaj.6030

Published online September 27, 2019

$* * *$

\section{Abstract}

Introduction: Very few population-based assessments of delirium have been performed to date. These have not assessed the implications of delirium after major surgical oncology procedures (MSOPs). We examined the temporal trends of delirium following 10 MSOPs, as well as patient and hospital delirium risk factors. Finally, we examined the effect of delirium on length of stay, in-hospital mortality, and hospital charges.

Methods: We retrospectively identified patients who underwent prostatectomy, colectomy, cystectomy, mastectomy, gastrectomy, hysterectomy, nephrectomy, oophorectomy, lung resection, or pancreatectomy within the Nationwide Inpatient Sample (2003-2013). We yielded a weighted estimate of 3431632 patients. Multivariable logistic regression (MLR) analyses identified the determinants of postoperative delirium, as well as the effect of delirium on length of stay, in-hospital mortality, and hospital charges.

Results: Between 2003 and 2013, annual delirium rate increased from 0.7 to 1.2\% (+6.0\%; $\mathrm{p}<0.001$ ). Delirium rates were highest after cystectomy (predicted probability [PP] 3.1\%) and pancreatectomy (PP 2.6\%) and lowest after prostatectomy (PP 0.15\%) and mastectomy (PP 0.13\%). Advanced age (odds ratio [OR] 3.80), maleness (OR 1.38), and higher Charlson comorbidity index (OR 1.20), as well as postoperative complications represent risk factors for delirium after MSOPs. Delirium after MSOP was associated with prolonged length of stay (OR 3.00), higher mortality (OR 1.15) and increased in-hospital charges (OR 1.13).

Conclusions: No contemporary population-based assessments of delirium after MSOP have been reported. According to our findings, delirium after MSOP has a profound impact on 
patient outcomes that ranges from prolonged length of stay to higher mortality and increased in-hospital charges.

\section{Introduction}

Delirium defined as an acute decline of cognition and attention, is a common and severe problem for hospitalized patients, especially in those who underwent surgical procedures. Institutional series demonstrated that delirium affects $10-31 \%$ of adult hospital admissions[1], and possibly is even more prevalent in surgical patients[2]. Additionally, delirium predisposes to greater morbidity, prolonged length of stay, higher mortality and increased in-hospital charges[3,4].

Institutional data showed that the odds of developing postoperative delirium are dependent on several factors, such as pre-existing cognitive dysfunction or pre-existing comorbidities[5]. Furthermore, others reported that incidence of delirium is variable according to patient characteristics and surgical procedures ${ }^{5}$. For example, Lee et al.[6] found a 13.8\% rate of delirium after cardiac surgery. Conversely, in a meta-analysis of 26 studies, Bruce et al.[7] observed a wide range of delirium rates after elective orthopedic surgery ranging from 3.6 to $28.3 \%$. Unfortunately, the variability of post-operative delirium rates was not examined in contemporary population-based studies. Specifically, no such study focused on delirium after major surgical oncology procedures (MSOPs). In consequence, neither its rates nor its consequences are known in MSOP setting.

To address the lack of data focusing on delirium after MSOPs, we assessed delirium temporal trends after ten MSOPs, namely prostatectomy, colectomy, cystectomy, mastectomy, gastrectomy, hysterectomy, nephrectomy, oophorectomy, lung resection and pancreatectomy. Moreover, we examined risk factors predisposing to post-operative delirium, as well as its association with length of stay, in-hospital mortality and hospital charges.

\section{Methods}

\section{Data source}

To assess the incidence of post-operative delirium after MSOPs, we relied on the NIS database (2003- 2013). The NIS is a set of longitudinal hospital inpatient databases included in the Healthcare Cost and Utilization Project family, created by the Agency for Healthcare Research and Quality through a Federal-state partnership[8]. The database includes 20\% of United States inpatient hospitalizations with discharge abstracts from 8 million hospital stays. It incorporates patient and hospital information, including Medicare, Medicaid, private insurance, and other insurance type patients.

\section{Study population}

Ten MSOPs were selected to serve the study purpose[9,10]: prostatectomy, colectomy, cystectomy, mastectomy, gastrectomy, hysterectomy, nephrectomy, oophorectomy, lung resection and pancreatectomy. Analyses were restricted to cancer diagnoses only. All procedures and diagnoses were coded using the International Classification of Disease, $9^{\text {th }}$ revision, Clinical Modification (ICD-9-CM) (Supplementary Table 1). 


\section{Outcomes of interest}

Administrative codes were utilized to identify delirium diagnosis as previously described[9], and were defined as the presence of one of nine ICD-9-CM diagnostic codes: alcohol withdrawal delirium (291.0), drug-induced delirium (292.81), presenile dementia with delirium (290.11), senile dementia with delirium (290.3), vascular dementia with delirium (290.41), subacute delirium (293.1), metabolic encephalopathy (348.31), toxic encephalopathy (349.82) or delirium not otherwise specified (293.0). Prolonged length of stay was defined as a hospitalization above the $75^{\text {th }}$ percentile for each examined MSOPs. Increased in-hospital charges were defined as amounts above the $75^{\text {th }}$ percentile for each of ten examined MSOPs.

\section{Patient and hospital characteristics}

Patient age, gender, race/ethnicity (Caucasian, African American and Others), Charlson comorbidity index (CCI)[11,12] and insurance status (private insurance, Medicare, Medicaid, and other [self-pay]) were defined according to NIS information. Ten previously reported[5,13-15] delirium risk factors such as dementia, alcohol-induced mental disorder, mood disorder, non-organic disorder, anxiety disorder, schizophrenia disorder, alcohol dependence, drug dependence syndrome, non-dependent drug use and drug induced disorder were tested in logistic regression models. Additional risk variables consisted of hospital region (Northeast, Midwest, South, West)[16], hospital size (small, medium and large) and hospital teaching vs. non-teaching status. Teaching institutions had an American Medical Association-approved residency program, were a member of the Council of Teaching Hospitals, or had a ratio of 0.25 or higher of full-time equivalent interns and residents to nonnursing home beds.[17] Lastly, annual MSOP hospital volume (low, medium and high), representing the number of MSOP performed at each participating institution during each study calendar year was calculated independently for each of the ten examined MSOPs[18]. Patients were divided according to 3 equal hospital volume tertiles, categorized as low-, medium-, and high-volume centers.

\section{Statistical analysis}

Data distribution was adjusted according to the provided NIS population weights to render estimates more accurate nationally. All analyses were performed on the weighted population.

First, medians and interquartile ranges, as well as frequencies and proportions were reported for continuous (age and length of stay) and categorical variables (gender, race, insurance status, CCI, annual MSOP hospital volume, region, hospital size, teaching status and rates of concomitant psychiatric diagnosed that are considered as established delirium risk factors[5,13-15]), respectively. The statistical significance of differences in medians and proportions was evaluated with the Kruskal-Wallis and chi-square tests.

Second, temporal trend rates were analyzed by the estimated annual percentage change (EAPC), which utilizes the linear regression methodology[19]. Third, five sets of separate multivariable logistic regression (MLR) models examined five specific end-points: 1) the first set of MLR models tested patient and hospital determinants of delirium after MSOPs, 2) the second set of MLR models tested the effect of post-operative complications (Supplementary Table 1) on delirium rates after MSOPs, 3) the third set of MLR models 
tested the effect of delirium on rates of prolonged ( $\geq 75^{\text {th }}$ percentile) length of stay, 4 ) the fourth set of MLR models tested the effect of delirium on rates of in-hospital mortality and 5) the fifth set of MLR models tested the effect of delirium on rates of increased $\left(\geq 75^{\text {th }}\right.$ percentile) in-hospital charges. Moreover, end-points 3) and 5) were subsequently individually re-examined for each MSOP. Specifically, the effect of delirium on prolonged length of stay and the effect of delirium on increased in-hospital charges were tested with a separate MLR for each of the ten examined MSOPs.

Finally, to adjust for clustering within hospitals, all five MVA regression models were fitted with generalized estimating equations[20]. Analyses were performed using the $\mathrm{R}$ software environment for statistical computing and graphics (version 3.3.0; http://www.rproject.org/).

\section{Results}

\section{General characteristics of the study populations}

Between 2003-2013, a weighted estimate of 3,431,632 patients underwent one of the ten examined MSOP. Overall, 1\% of patients were discharged with the diagnosis of delirium. Patients with delirium were more frequently older ( 75 vs. 64 years), male (55.9 vs $44.5 \%$ ), Caucasian (69.1 vs. 62.8\%), Medicare insured (78.3 vs. 46\%) and exhibited higher CCI (CCI $\geq 2$ : 23.8 vs. $11.2 \%$ ) than controls. Non-teaching hospitals status (43.1 vs. $41.5 \%$ ) and lowest hospital surgical volume tertile accounted for higher rates of delirium (35.2 vs. 32.5\%). Moreover, length of stay was longer (10 vs. 4 days), when delirium was diagnosed (Table 1). The proportion of patients with CCI $\geq 1$ increased from 33.8 to $39.8 \%$ (EAPC: $+1.7 \%$, 95\%CI: 1.41-2.03, $\mathrm{p}<0.0001$ ), during the study period.

\section{Temporal trend analyses}

Between 2003 to 2013 (Figure 1), the annual delirium rate increased from 0.7 to $1.2 \%$ (EAPC: $+6.0 \%$, CI: +3.6 to +8.5, $\mathrm{p}<0.001$ ). Within individual MSOPs, colectomy (EAPC: $+7.2 \%$ ) and pancreatectomy (EAPC: $+6.5 \%$ ) exhibited the highest increase in the annual rate of delirium compared to prostatectomy (EAPC: -1.68), which exhibited the lowest rate (Supplementary Figure 1).

\section{Multivariable logistic regression models testing for patient and hospital determinants of delirium after MSOPs}

According to multivariable predicted probability (PP) of delirium after MSOPs (Figure 2), the highest rate was recorded after cystectomy (PP: 3.1\%, standard deviation [SD]: 0.03), followed by pancreatectomy (PP: 2.6\%, SD: 0.03) and gastrectomy (PP: 2.3\%, SD: 0.04). The lowest rates of postoperative delirium were recorded after prostatectomy (PP: 0.15\%, SD: 0.001) and mastectomy (PP: 0.13\%, SD: 0.001).

Patient risk factors associated with delirium after MSOPs were older age (55-64 years odds ratio [OR]: 1.90, $\mathrm{p}<0.0001 ; \geq 65$ years OR: 3.80, $\mathrm{p}<0.0001$ ) and male gender (OR: 1.38, $\mathrm{p}<0.0001$ ). CCI score $\geq 1$ ( 1 OR: $1.07, \mathrm{p}=0.03$; $\geq 2$ OR: $1.20, \mathrm{p}<0.001$ ) resulted in a marginal increase. Medicaid (OR: 1.21, $\mathrm{p}=0.02$ ) and Medicare (OR: 1.58, $\mathrm{p}<0.0001$ ) insurance status also increased the delirium rates, relative to private insurance status. Finally, African 
American (OR: 0.82, $\mathrm{p}=0.0002$ ) and non-Caucasian race (OR: 0.88, $\mathrm{p}=0.0001$ ) were associated with lower delirium rates after MSOPs, relative to Caucasian patients.

Of ten established delirium risk factors, seven achieved independent predictor status (Supplementary Table 2): dementia (OR: 24.07, $\mathrm{p}<0.0001$ ), alcohol dependence (OR: 14.51, $\mathrm{p}<0.0001$ ), drug-induced disorder (OR: 4.81, $\mathrm{p}<0.0001$ ), mood disorder (OR: 2.43, $\mathrm{p}<0.0001$ ), drug dependence syndrome (OR: $1.70 \mathrm{p}=0.02)$, anxiety disorder (OR: 1.53, $\mathrm{p}<0.0001$ ) and non-dependent drugs use (OR: 1.11, $\mathrm{p}=0.01)$.

\section{Multivariable logistic regression models testing the effect of any post-operative complications on delirium after MSOPs}

According to presence or absence of delirium after MSOPs, complication rates ranged as follows: intraoperative (3.3 vs. 1.7\%), respiratory (38.7 vs. 9.8\%), neurological (1.5 vs. $0.7 \%$ ), infectious (14.7 vs. $2.6 \%$ ), vascular (7.8 vs. $2.1 \%$ ), gastrointestinal (27.0 vs. $11.2 \%$ ), cardiac (17.0 vs. $5.8 \%)$, genitourinary (10.9 vs. $5.5 \%)$, wound (10.0 vs. $2.4 \%)$ and other (33.2 vs. $10.1 \%)$. Of ten examined postoperative complications, seven achieved independent predictor status (Table 2): respiratory (OR: 1.92, $\mathrm{p}<0.0001$ ), other (OR: 1.51, $\mathrm{p}<0.0001$ ), neurological (OR: 1.46, $\mathrm{p}=0.0005$ ), infectious (OR: 1.36, $\mathrm{p}<0.0001$ ), vascular (OR: 1.27, $\mathrm{p}<0.0001$ ), gastrointestinal (OR: 1.14, $\mathrm{p}<0.0001$ ) and cardiac (OR: 1.10, $\mathrm{p}=0.008$ ). According to presence or absence of delirium after MSOPs, post-MSOPs transfusion rates were $28.7 \mathrm{vs.}$ $11.5 \%$, respectively. Postoperative transfusions (OR: $1.33, \mathrm{p}<0.0001)$ also increased the delirium rate.

Multivariable logistic regression models testing the effect of delirium on prolonged $(\geq 75$ th percentile) length of stay

Stratification according to presence or absence of delirium after MSOPs was associated with prolonged ( $\geq 75^{\text {th }}$ percentile) length of stay (OR: 3.00, 95\%CI: $2.75-3.24, p<0.0001$ ). In all ten MSOP-specific models examining the length of stay above the $75^{\text {th }}$ percentile, delirium achieved independent predictor status (Table 3): mastectomy (OR: 11.34, $\mathrm{p}<0.0001$ ), prostatectomy (OR: 10.45, $\mathrm{p}<0.0001$ ), hysterectomy (OR: 5.99, $\mathrm{p}<0.0001$ ), nephrectomy (OR: 4.54, $\mathrm{p}<0.0001$ ), oophorectomy (OR: 3.54, $\mathrm{p}<0.0001$ ), lung resection (OR: 2.55, $\mathrm{p}<0.0001$ ), cystectomy (OR: $2.03, \mathrm{p}<0.0001$ ), gastrectomy (OR: $1.88, \mathrm{p}<0.0001$ ), colectomy (OR: 1.86, $\mathrm{p}<0.0001)$ and pancreatectomy (OR: 1.84, $\mathrm{p}<0.0001)$.

\section{Multivariable logistic regression models testing the effect of delirium on in-hospital mortality}

Overall, 1.1\% of MSOP patients died during the hospitalization. Stratification according to presence or absence of delirium after MSOPs was associated with higher (6 vs. 1\%, $\mathrm{p}<0.0001)$ rate of in-hospital mortality. Moreover, delirium after MSOPs was associated with 1.15-fold (OR: 1.15, 95\% CI: 1.10-1.21, p<0.0001) mortality rate increase. Low absolute mortality rates after individual MSOPs (ranging from 0.13 to 3.2\%), prevented the reporting of multivariable MSOP specific mortality rates after delirium. 


\section{Multivariable logistic regression models testing the effect of delirium on increased $(\geq 75$ th percentile) in-hospital charges}

Stratification according to presence or absence of delirium after MSOPs was associated with increased ( $\geq 75^{\text {th }}$ percentile) in-hospital charges (OR: 1.13 , 95\%CI: 1.05-1.21, $p=0.001$ ). In all ten MSOP-specific models examining the hospital charges above the $75^{\text {th }}$ percentile, delirium achieved independent predictor status (Table 4): prostatectomy (OR: 2.04, $\mathrm{p}<0.0001$ ), lung resection (OR: 2.02, $\mathrm{p}<0.0001$ ), oophorectomy (OR: 1.99, $\mathrm{p}<0.0001$ ), cystectomy (OR: 1.86, $\mathrm{p}<0.0001$ ), hysterectomy (OR: 1.74, $\mathrm{p}<0.0001$ ), nephrectomy (OR: 1.74, $\mathrm{p}<0.0001$ ), gastrectomy (OR: 1.73, $\mathrm{p}<0.0001$ ), pancreatectomy (OR: 1.64, $\mathrm{p}<0.0001$ ), colectomy (OR: 1.59, $\mathrm{p}<0.0001)$ and mastectomy (OR: 1.49, $\mathrm{p}<0.0001)$.

\section{Discussion}

Postoperative delirium is a multi-faceted problem that is associated with poor perioperative outcomes, increased long-term adverse sequelae and a significant cost burden ${ }^{19}$. To date, several single and multi-institutional series have assessed the rate of delirium and its implication on patient outcomes. Nonetheless, contemporary population-based assessments of delirium using large administrative databases are lacking. Based on these considerations, we sought to examine population-level trends in delirium following MSOP. To accomplish this task, we relied on the NIS database including patients underwent one of ten MSOPs. Our study yielded several noteworthy findings.

First, our population-based analyses revealed a rate of delirium after MSOPs of $1 \%$. This finding contrasts with the postoperative delirium incidence currently reported in the literature, ranging from $4 \%$ for minor procedures, to $35-65 \%$ for major procedures[13]. It should be noted that delirium rates may be captured less frequently than other comorbidities such as infection[21], venous thromboembolism[22] or common postoperative complications[9]. Moreover, delirium may go unnoticed in older patients with other comorbidities that are given greater importance in the acute care setting such as after MSOPs. Last but not least, delirium may go unnoticed because of absence of associated symptoms. In that regard, Lipowski et al.[23] distinguished between hypoactive (quiet) and hyperactive variants of delirium. Patients with active delirium are easily identifiable. Conversely, individuals with hypoactive variant, which accounts for $75 \%$ of cases, may permanently go unnoticed[24]. In consequence, prospective studies that à priori include delirium among outcomes of interest may contrast with population-based analyses, where delirium rates may be underreported.

Second, we identified an increase in delirium rates after MSOP, over time. Several factors may explain this phenomenon. For example, increasing comorbidities of patients undergoing MSOP may have contributed to these findings. Indeed, we found that the annual rate of CCI $\geq 1$ increased by $1.7 \%$ (95\%CI: $1.41-2.03, \mathrm{p}<0.0001)$ during the study period.

Third, to the best of our knowledge we are first to report delirium rates after specific MSOPs. Here, cystectomy (3.2\%), pancreatectomy (2.6\%) and gastrectomy (2.4\%) demonstrated the highest rates of delirium. Conversely, two procedures namely prostatectomy $(0.14 \%)$ and mastectomy $(0.13 \%)$ were associated with significantly lower rates than average (1\%). These rates suggest a relationship between post-MSOP delirium and the complexity of 
the surgical procedures. For example, cystectomy and pancreatectomy are associated with longer operative times and higher use of analgesia. Both procedures respectively represent the first highest and the second highest, when delirium rates are considered.

Fourth, analyses of established delirium risk factors validated our findings. Specifically, well-known[13,25] delirium risk factors such as dementia (OR: 24.07), alcohol dependence (OR: 14.51) and mood disorder (2.43) all strongly and significantly increased delirium rates after MSOPs. Moreover, drug-induced disorder, anxiety disorder and drug dependence syndrome were also strongly associated with delirium, which is often reported in patients treated with opioids, benzodiazepines and sedatives[15].

Fifth, to the best of our knowledge, we are first to examine the association between delirium after MSOPs and specific complications. Here, of ten examined postoperative complications, seven achieved independent predictor status. Specifically, respiratory, other, neurological, infectious, vascular, gastrointestinal and cardiac complications were associated with higher rates of post MSOP delirium. To the best of our knowledge, we are also first to report that post-operative transfusions were associated with increased rate of delirium (OR: 1.33). This result is worrisome since approximately 30\% MSOP patients require a transfusion during hospital stay[26].

Sixth, our findings suggest that length of stay is longer in patients experiencing delirium after MSOPs. Based on our observations, the effect of delirium on length of stay above the $75^{\text {th }}$ percentile is strongest for MSOPs with short length of stay. Conversely, MSOP-specific delirium rates are weakest for MSOPs with long length of stay. For example, after prostatectomy (median length of stay 2 days) and mastectomy (median length of stay 2 days) the effect of delirium was 10-fold higher. Conversely, after cystectomy (median length of stay 8 days) or colectomy (median length of stay 7 days) the effect of delirium was only 2fold higher. These observations contrast with the absolute risk of delirium. The latter is lowest for procedures with shortened length of stay and highest for procedures with prolonged length of stay. Similarly, we also observed a similar relationship between delirium and increased inhospital charges.

Last but not least, we are first to report a relationship between delirium and higher inhospital mortality after MSOPs. Overall, patients who experienced delirium had a 15\% increase of in-hospital mortality compared to those without delirium. These findings are worrisome and they should motivate physician toward a higher delirium monitoring in patients treated with one of the ten MSOPs.

Finally, from a stricter urological point of view, it can be postulated that this analysis may be of interest for urologists, who are dealing with complex patients with multiple urological and non-urological issues who may benefit from a multidisciplinary evaluation. Nowadays indeed, surgical indications are evolving towards a more aggressive behavior including radical surgery in advanced urological malignancies [27]and, or metastasectomy in oligometatstaic cancers[28]. In this scenario, urologist must be awarded of possible complications surrounding MSOPs, in order to reduce and possibly prevent those in their patients[10,18]. Last but not least, it should be noted that post-operative delirium might be easily prevented following evidence-based approach. On the other hand, our data emphasized that post-operative delirium is still a relatively frequent event Iin our daily practice. In 
consequence, our data may be useful for shed a light on this issue improving our clinical practice

Our study is not devoid of limitations, which apply to all studies with retrospective designs. Additionally, our study was unable to adjust for tumor characteristics and longitudinal data was also unavailable. Moreover, we were unable to control for some risk factors, such as laboratory values and baseline cognitive impairment. Finally, the NIS database does not specify delirium type (hyperactive vs. hypoactive), which could not be determine. Despite these limitations, we were able to provide new insight about the importance of delirium after MSOPs.

\section{Conclusions}

No contemporary population-based assessments of delirium after MSOP have been reported. According to our findings, delirium after MSOP has a profound impact on patient outcomes that ranges from prolonged length of stay to higher mortality and increased in-hospital charges. 


\section{References}

1. Siddiqi N, House AO, Holmes JD. Occurrence and outcome of delirium in medical inpatients: a systematic literature review. Age Ageing 2006;35:350-64. doi:10.1093/ageing/afl005.

2. Raats JW, van Eijsden WA, Crolla RMPH, Steyerberg EW, van der Laan L. Risk Factors and Outcomes for Postoperative Delirium after Major Surgery in Elderly Patients. PloS One 2015;10:e0136071. doi:10.1371/journal.pone.0136071.

3. Leslie DL, Zhang Y, Bogardus ST, Holford TR, Leo-Summers LS, Inouye SK. Consequences of preventing delirium in hospitalized older adults on nursing home costs. J Am Geriatr Soc 2005;53:405-9. doi:10.1111/j.1532-5415.2005.53156.x.

4. Leslie DL, Zhang Y, Holford TR, Bogardus ST, Leo-Summers LS, Inouye SK. Premature death associated with delirium at 1-year follow-up. Arch Intern Med 2005;165:1657-62. doi:10.1001/archinte.165.14.1657.

5. Robinson TN, Raeburn CD, Tran ZV, Angles EM, Brenner LA, Moss M. Postoperative delirium in the elderly: risk factors and outcomes. Ann Surg 2009;249:173-8. doi:10.1097/SLA.0b013e31818e4776.

6. Lee A, Mu JL, Joynt GM, Chiu CH, Lai VKW, Gin T, et al. Risk prediction models for delirium in the intensive care unit after cardiac surgery: a systematic review and independent external validation. Br J Anaesth 2017;118:391-9. doi:10.1093/bja/aew476.

7. Bruce AJ, Ritchie CW, Blizard R, Lai R, Raven P. The incidence of delirium associated with orthopedic surgery: a meta-analytic review. Int Psychogeriatr 2007;19:197-214. doi:10.1017/S104161020600425X.

8. HCUP-US NIS Overview n.d. https://www.hcup-us.ahrq.gov/nisoverview.jsp (accessed July 20, 2017).

9. Tan H-J, Saliba D, Kwan L, Moore AA, Litwin MS. Burden of Geriatric Events Among Older Adults Undergoing Major Cancer Surgery. J Clin Oncol Off J Am Soc Clin Oncol 2016;34:1231-8. doi:10.1200/JCO.2015.63.4592.

10. Nazzani S, Preisser F, Mazzone E, Tian Z, Mistretta FA, Shariat SF, et al. In-hospital length of stay after major surgical oncological procedures. Eur J Surg Oncol J Eur Soc Surg Oncol Br Assoc Surg Oncol 2018;44:969-74. doi:10.1016/j.ejso.2018.05.001.

11. Charlson ME, Pompei P, Ales KL, MacKenzie CR. A new method of classifying prognostic comorbidity in longitudinal studies: development and validation. J Chronic Dis 1987;40:373-83.

12. Deyo RA, Cherkin DC, Ciol MA. Adapting a clinical comorbidity index for use with ICD-9-CM administrative databases. J Clin Epidemiol 1992;45:613-9.

13. Rudolph JL, Marcantonio ER. Review articles: postoperative delirium: acute change with long-term implications. Anesth Analg 2011;112:1202-11. doi:10.1213/ANE.0b013e3182147f6d.

14. Litaker D, Locala J, Franco K, Bronson DL, Tannous Z. Preoperative risk factors for postoperative delirium. Gen Hosp Psychiatry 2001;23:84-9.

15. Lin RY, Heacock LC, Fogel JF. Drug-induced, dementia-associated and nondementia, non-drug delirium hospitalizations in the United States, 1998-2005: an analysis of the national inpatient sample. Drugs Aging 2010;27:51-61. doi:10.2165/11531060-000000000-00000.

16. Bureau UC. Census.gov n.d. https://www.census.gov/en.html (accessed December 15, 2017). 
17. NIS Description of Data Elements n.d. https://www.hcupus.ahrq.gov/db/nation/nis/nisdde.jsp (accessed July 21, 2017).

18. Nazzani S, Bandini M, Preisser F, Mazzone E, Marchioni M, Tian Z, et al. Postoperative paralytic ileus after major oncological procedures in the enhanced recovery after surgery era: A population based analysis. Surg Oncol 2019;28:201-7. doi:10.1016/j.suronc.2019.01.011.

19. Ghani KR, Sammon JD, Karakiewicz PI, Sun M, Bhojani N, Sukumar S, et al. Trends in surgery for upper urinary tract calculi in the USA using the Nationwide Inpatient Sample: 1999-2009. BJU Int 2013;112:224-30. doi:10.1111/bju.12059.

20. Panageas KS, Schrag D, Riedel E, Bach PB, Begg CB. The effect of clustering of outcomes on the association of procedure volume and surgical outcomes. Ann Intern Med 2003;139:658-65.

21. Sammon JD, Klett DE, Sood A, Olugbade K, Schmid M, Kim SP, et al. Sepsis after major cancer surgery. J Surg Res 2015;193:788-94. doi:10.1016/j.jss.2014.07.046.

22. Trinh VQ, Karakiewicz PI, Sammon J, Sun M, Sukumar S, Gervais M-K, et al. Venous thromboembolism after major cancer surgery: temporal trends and patterns of care. JAMA Surg 2014;149:43-9. doi:10.1001/jamasurg.2013.3172.

23. Lipowski ZJ. Transient cognitive disorders (delirium, acute confusional states) in the elderly. Am J Psychiatry 1983;140:1426-36. doi:10.1176/ajp.140.11.1426.

24. Marcantonio E, Ta T, Duthie E, Resnick NM. Delirium severity and psychomotor types: their relationship with outcomes after hip fracture repair. J Am Geriatr Soc 2002;50:850-7.

25. Grover S, Ghormode D, Ghosh A, Avasthi A, Chakrabarti S, Mattoo SK, et al. Risk factors for delirium and inpatient mortality with delirium. J Postgrad Med 2013;59:263-70. doi:10.4103/0022-3859.123147.

26. Ecker BL, Simmons KD, Zaheer S, Poe S-LC, Bartlett EK, Drebin JA, et al. Blood Transfusion in Major Abdominal Surgery for Malignant Tumors: A Trend Analysis Using the National Surgical Quality Improvement Program. JAMA Surg 2016;151:518-25. doi:10.1001/jamasurg.2015.5094.

27. Nini A, Capitanio U, Larcher A, Dell’Oglio P, Dehò F, Suardi N, et al. Perioperative and Oncologic Outcomes of Nephrectomy and Caval Thrombectomy Using Extracorporeal Circulation and Deep Hypothermic Circulatory Arrest for Renal Cell Carcinoma Invading the Supradiaphragmatic Inferior Vena Cava and/or Right Atrium. Eur Urol 2018;73:793-9. doi:10.1016/j.eururo.2017.08.019.

28. Ciriaco P, Briganti A, Bernabei A, Gandaglia G, Carretta A, Viola C, et al. Safety and Early Oncologic Outcomes of Lung Resection in Patients with Isolated Pulmonary Recurrent Prostate Cancer: A Single-center Experience. Eur Urol 2019;75:871-4. doi:10.1016/j.eururo.2018.12.029. 


\section{Figures and Tables}

Fig. 1. Overall delirium frequency following major surgical oncology procedures (MSOPs) in 3431632 patients, Nationwide Inpatient Sample, 2003-2013.

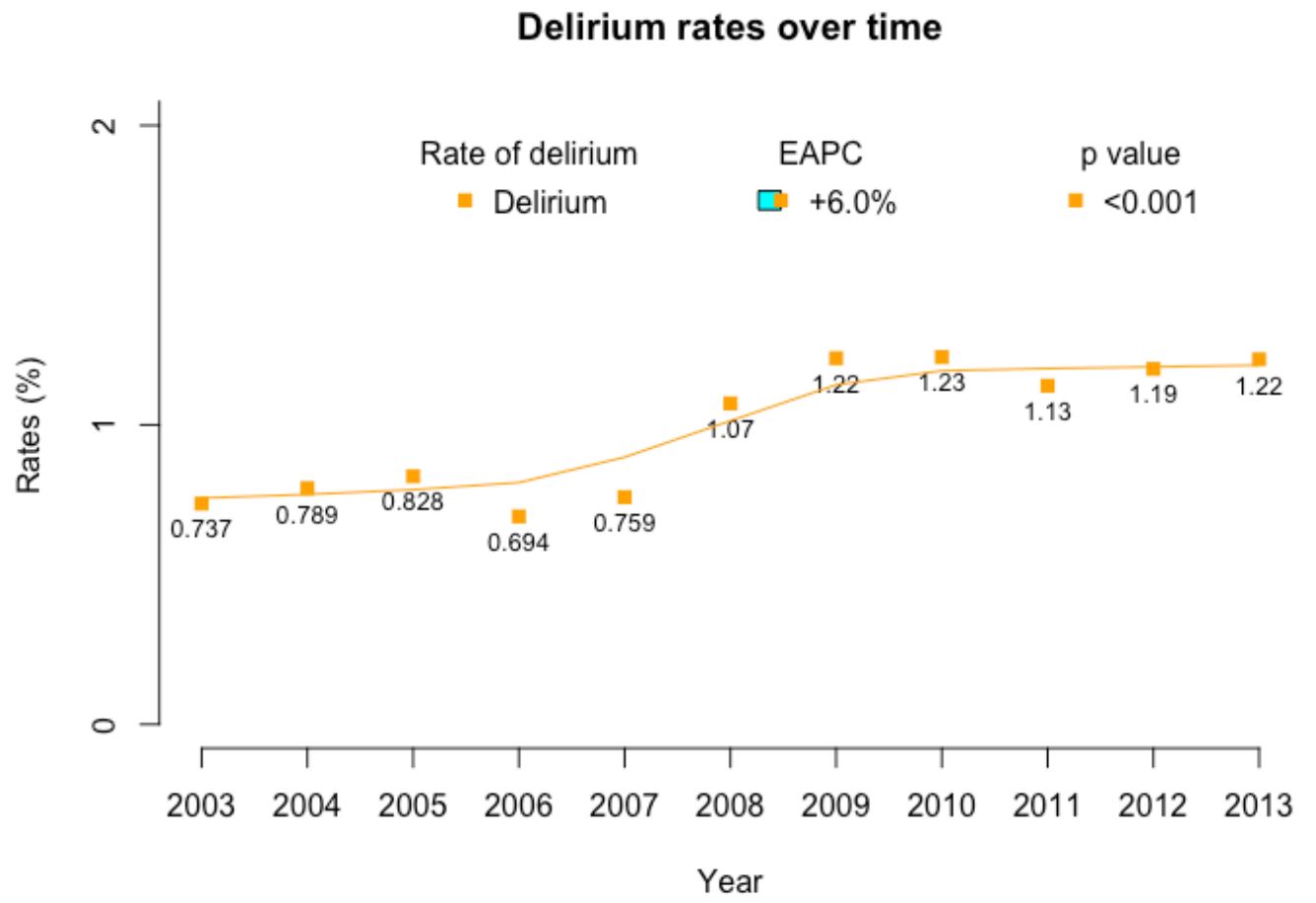


Fig. 2. Model-adjusted probability of a delirium event according to major surgical oncology procedures (MSOPs). Predicted probabilities are derived from multivariable

logistic regression models that adjusted for patient demographics (ie, gender, race/ethnicity, Charlson comorbidity index and insurance status), and hospital characteristics (ie, hospital size, location, length of stay, teaching status, region, and hospital MSOP volume), as well as for patients neurologic disorder (dementia, alcohol induced mental disorder, mood disorder, non-organic disorder, anxiety disorder, alcohol dependence, schizophrenia disorder, drug dependence syndrome, non-dependent drug use and drug induced disorder).

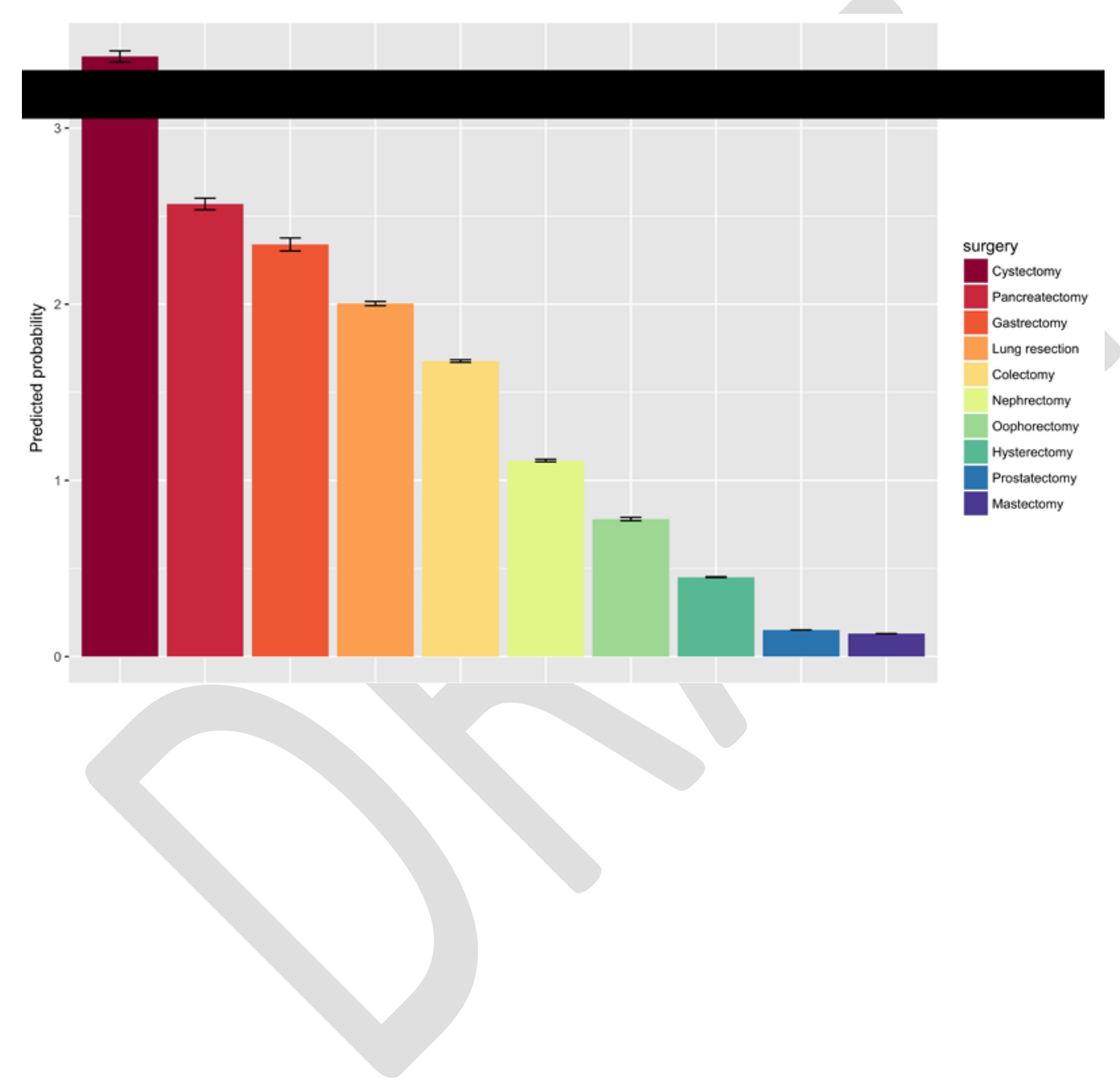




\begin{tabular}{|c|c|c|c|}
\hline Variables & Overall (\%) & $\begin{array}{c}\text { Without delirium } \\
(\%)\end{array}$ & $\begin{array}{c}\text { With delirium } \\
(\%)\end{array}$ \\
\hline Weighted no. (\%) of patients & $3431632(100.0)$ & 3398637 (99.04) & $32994(0.96)$ \\
\hline Age at surgery, median (IQR) & $64(56-73)$ & $64(56-73)$ & $75(67-81)$ \\
\hline Length of stay, median (IQR) & $4(2-7)$ & $4(2-7)$ & $10(7-16)$ \\
\hline \multicolumn{4}{|l|}{ Year of surgery } \\
\hline $2003-2008$ & 61.2 & 61.3 & 51.7 \\
\hline $2009-2013$ & 38.8 & 38.7 & 48.3 \\
\hline \multicolumn{4}{|l|}{ Gender } \\
\hline Female & 55.4 & 55.5 & 44.1 \\
\hline Male & 44.6 & 44.5 & 55.9 \\
\hline \multicolumn{4}{|l|}{ Race } \\
\hline Caucasian & 62.9 & 62.8 & 69.1 \\
\hline African American & 8 & 8 & 6.3 \\
\hline Non-Caucasian & 29.1 & 29.2 & 24.7 \\
\hline \multicolumn{4}{|l|}{ CCI } \\
\hline 0 & 63.6 & 63.8 & 44.8 \\
\hline 1 & 25 & 25 & 31.3 \\
\hline$\geq 2$ & 11.3 & 11.2 & 23.8 \\
\hline \multicolumn{4}{|l|}{ Hospital teaching status } \\
\hline Non-teaching & 41.5 & 41.5 & 43.1 \\
\hline Teaching & 58.5 & 58.5 & 56.9 \\
\hline \multicolumn{4}{|l|}{$\begin{array}{l}\text { Annual MSOP hospital } \\
\text { volume }\end{array}$} \\
\hline Low & 32.5 & 32.5 & 35.2 \\
\hline Medium & 33.8 & 33.8 & 34.8 \\
\hline High & 33.7 & 33.7 & 30.0 \\
\hline \multicolumn{4}{|l|}{ Hospital region } \\
\hline South & 35.6 & 35.6 & 34 \\
\hline Midwest & 23.5 & 23.5 & 26.9 \\
\hline Northeast & 21.6 & 21.6 & 20.8 \\
\hline West & 19.3 & 19.3 & 18.2 \\
\hline \multicolumn{4}{|l|}{ Insurance status } \\
\hline Private & 44.1 & 44.4 & 16.2 \\
\hline Medicaid & 4.8 & 4.9 & 3.3 \\
\hline Medicare & 46.3 & 46 & 78.3 \\
\hline Other & 4.7 & 4.8 & 2.3 \\
\hline Hospital size & & & \\
\hline
\end{tabular}




\begin{tabular}{|c|c|c|c|}
\hline Large & 68.4 & 68.4 & 69.6 \\
\hline Medium & 21.5 & 21.5 & 20.8 \\
\hline Small & 10.1 & 10.1 & 9.5 \\
\hline \multicolumn{4}{|l|}{ MSOP } \\
\hline Prostatectomy & 19.8 & 20 & 2.8 \\
\hline Colectomy & 18.8 & 18.7 & 33 \\
\hline Cystectomy & 2.6 & 2.6 & 8.5 \\
\hline Gastrectomy & 2.2 & 2.2 & 5.3 \\
\hline Hysterectomy & 15.5 & 15.6 & 7.2 \\
\hline Mastectomy & 15.6 & 15.7 & 2.1 \\
\hline Nephrectomy & 11 & 11 & 12.6 \\
\hline Oophorectomy & 2.2 & 2.2 & 1.7 \\
\hline Pancreatectomy & 2 & 2 & 5.3 \\
\hline Lung resection & 10.3 & 10.2 & 21.4 \\
\hline \multicolumn{4}{|l|}{$\begin{array}{l}\text { Concomitant psychiatric } \\
\text { diagnoses }\end{array}$} \\
\hline Dementia & 0.1 & 0.04 & 1.9 \\
\hline $\begin{array}{l}\text { Alcohol induced mental } \\
\text { disorder }\end{array}$ & 0.1 & 0.1 & 1.3 \\
\hline Mood disorder & 0.8 & 0.8 & 2.4 \\
\hline Non-organic disorder & 0.2 & 0.2 & 0.7 \\
\hline Anxiety disorder & 5 & 5 & 7.2 \\
\hline Alcohol dependence & 0.3 & 0.3 & 0.5 \\
\hline Schizophrenia disorder & 0.5 & 0.4 & 7.1 \\
\hline Drug dependence syndrome & 0.1 & 0.1 & 0.5 \\
\hline Non-dependent drug use & 10.4 & 10.3 & 12.6 \\
\hline Drug induced disorder & 0.1 & 0.1 & 1 \\
\hline
\end{tabular}

CCI: Charlson comorbidity index; IQR: interquartile rage; MSOP: major surgical oncology procedures. 


\begin{tabular}{|c|c|c|c|c|}
\hline Complications & OR & CI $2.50 \%$ & CI $97.50 \%$ & $\mathbf{p}$ \\
\hline Respiratory & 1.92 & 1.80 & 2.04 & $<0.0001$ \\
\hline Other & 1.51 & 1.42 & 1.60 & $<0.0001$ \\
\hline Neurological & 1.46 & 1.18 & 1.80 & 0.0005 \\
\hline Infectious & 1.36 & 1.22 & 1.51 & $<0.0001$ \\
\hline Transfusions & 1.33 & 1.25 & 1.41 & $<0.0001$ \\
\hline Vascular & 1.27 & 1.15 & 1.40 & $<0.0001$ \\
\hline Gastrointestinal & 1.14 & 1.07 & 1.22 & $<0.0001$ \\
\hline Cardiac & 1.10 & 1.03 & 1.18 & 0.008 \\
\hline Intraoperative & 1.10 & 0.96 & 1.28 & 0.2 \\
\hline Genitourinary & 1.06 & 0.97 & 1.16 & 0.2 \\
\hline Wound infections & 1.01 & 0.90 & 1.14 & 0.8 \\
\hline
\end{tabular}

Analysis was adjusted for type of surgery, length of stay, age, gender, race, type of procedure, year of surgery, region of the hospital, teaching status, hospital size, annual MSOP hospital volume, Charlson comorbidity index, and insurance status. CI: confidence interval; OR: odds ratio. 


\begin{tabular}{|c|c|c|c|c|c|}
\hline $\begin{array}{l}\text { Table 3. Multivariable logist } \\
\text { procedure, year of surgery, } \\
\text { volume, hospital size, Charl } \\
\text { predicting the effect of delir } \\
\text { patients, Nationwide Inpatie }\end{array}$ & $\begin{array}{l}\text { regres } \\
\text { ion of } \\
\text { como } \\
\text { on el } \\
\text { Sampl }\end{array}$ & $\begin{array}{l}\text { models } \\
\text { hospita } \\
\text { ity inde } \\
\text { ed leng } \\
03-201\end{array}$ & $\begin{array}{l}\text { er fittin } \\
\text { aching } s \\
\text { isuranc } \\
\text { stay }(\geq\end{array}$ & $\begin{array}{l}\text { r age, ge } \\
\text { is, annua } \\
\text { tus, and } \\
\text { h percen }\end{array}$ & $\begin{array}{l}\text { ler, race, type of } \\
\text { MSOP hospital } \\
\text { omplications, for } \\
\text { e) in } 3431 \text { MSOP }\end{array}$ \\
\hline $\begin{array}{l}\text { Procedure specific effect of } \\
\text { delirium on length of stay } \\
\text { higher than the } 75^{\text {th }} \\
\text { percentile }\end{array}$ & OR & $\begin{array}{c}\text { CI } \\
2.50 \%\end{array}$ & $\begin{array}{c}\text { CI } \\
97.50 \%\end{array}$ & $\mathbf{p}$ & $\begin{array}{l}\text { Overall median length } \\
\text { of stay, days (IQR) }\end{array}$ \\
\hline Mastectomy & 11.34 & 8.26 & 16.04 & $<0.0001$ & $2(1-2)$ \\
\hline Prostatectomy & 10.45 & 8.13 & 13.57 & $<0.0001$ & $2(1-3)$ \\
\hline Hysterectomy & 5.99 & 5.35 & 6.73 & $<0.0001$ & $3(2-5)$ \\
\hline Nephrectomy & 4.54 & 4.19 & 4.92 & $<0.0001$ & $4(3-6)$ \\
\hline Oophorectomy & 3.54 & 2.83 & 4.42 & $<0.0001$ & $4(2-7)$ \\
\hline Lung resection & 2.55 & 2.41 & 2.69 & $<0.0001$ & $6(4-9)$ \\
\hline Cystectomy & 2.03 & 1.85 & 2.23 & $<0.0001$ & $8(7-11)$ \\
\hline Gastrectomy & 1.88 & 1.67 & 2.11 & $<0.0001$ & $10(8-16)$ \\
\hline Colectomy & 1.86 & 1.78 & 1.95 & $<0.0001$ & $7(5-11)$ \\
\hline Pancreatectomy & 1.84 & 1.64 & 2.06 & $<0.0001$ & $10(7-16)$ \\
\hline
\end{tabular}

CI: confidence interval; IQR: interquartile range; OR: odds ratio,

Table 4. Multivariable logistic regression models, after fitting for age, gender, race, type of procedure, year of surgery, region of the hospital, teaching status, Annual MSOP hospital volume, hospital size, Charlson comorbidity index, insurance status and complications, for predicting the effect of delirium on increased in-hospital charges $(\geq 75$ th percentile) in 3431632 MSOP patients, Nationwide Inpatient Sample, 2003-2013

\begin{tabular}{|l|c|c|c|c|}
\hline $\begin{array}{l}\text { Procedure specific effect of delirium on } \\
\text { hospital charges higher than the 75th } \\
\text { percentile }\end{array}$ & OR & CI 2.50\% & CI 97.50\% & p \\
\hline Prostatectomy & 2.04 & 1.76 & 2.36 & $<0.0001$ \\
\hline Lung resection & 2.02 & 1.91 & 2.13 & $<0.0001$ \\
\hline Oophorectomy & 1.99 & 1.63 & 2.42 & $<0.0001$ \\
\hline Cystectomy & 1.86 & 1.70 & 2.03 & $<0.0001$ \\
\hline Hysterectomy & 1.77 & 1.61 & 1.95 & $<0.0001$ \\
\hline Nephrectomy & 1.74 & 1.62 & 1.87 & $<0.0001$ \\
\hline Gastrectomy & 1.73 & 1.54 & 1.94 & $<0.0001$ \\
\hline Pancreatectomy & 1.64 & 1.46 & 1.84 & $<0.0001$ \\
\hline Colectomy & 1.59 & 1.52 & 1.66 & $<0.0001$ \\
\hline Mastectomy & 1.49 & 1.23 & 1.80 & $<0.0001$ \\
\hline
\end{tabular}

CI: confidence interval; OR: odds ratio. 
Supplementary Fig. 1. Delirium frequency of 3,431,632 patients underwent one of the ten examined major surgical oncology procedures (MSOPs), Nationwide Inpatient Sample, 20032013.

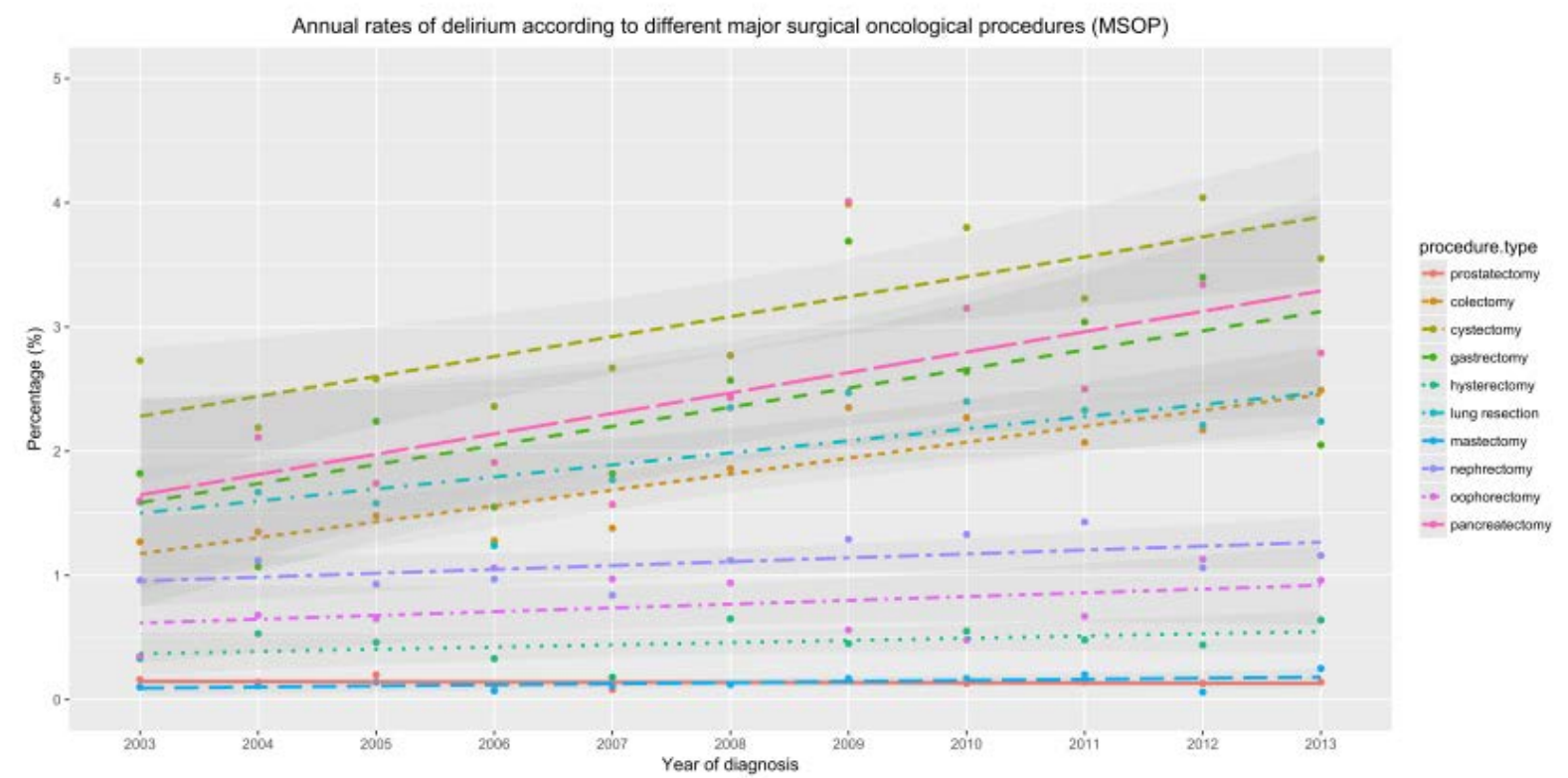

\begin{tabular}{|c|c|}
\hline \multicolumn{2}{|c|}{$\begin{array}{l}\text { Supplementary Table 1. Nationwide inpatient sample codes for major surgical oncological } \\
\text { procedure (MSOP) and complications }\end{array}$} \\
\hline MSOP & Codes \\
\hline Cystectomy & "576","577","688" \\
\hline Pancreatectomy & "604","605","6062" \\
\hline Lung resection & "3220","3229","323","324","325","326","329" \\
\hline Gastrectomy & "435","436","437","438","439" \\
\hline Prostatectomy & "525","526","527" \\
\hline Nephrectomy & "554","5551","5552","5554" \\
\hline Hysterectomy & "683","684","685","686","687","688","689" \\
\hline Oophorectomy & "653","654","655","656" \\
\hline Mastectomy & "8521","8522","8523","854" \\
\hline Colectomy & "457","458","484","485","486","173" \\
\hline Complications & Codes \\
\hline Intraoperative & "9982" \\
\hline Cardiac & $\begin{array}{l}\text { "4100","4101","4102","4103","4104","4105","4106","4107","410 } \\
\text { 8","4109","4110","4111","41181","41189","40201","40211","402 } \\
\text { 91","4280","4281","42821","42831","42841","4289","4275","997 } \\
\text { 1" }\end{array}$ \\
\hline Respiratory & $\begin{array}{c}\text { "5180","5184","5185","5187","51881","514","4660","46611","46 } \\
\text { 619","4800","4801","4802","4803","4808","4809","481","4820"," } \\
\text { 4821",,"4822","4823","4824",,"4828","4829","5070","51881", } \\
\text { "4830","4831","4838","485","486","7991","9973" }\end{array}$ \\
\hline
\end{tabular}




\begin{tabular}{|c|c|}
\hline Genitourinary & $\begin{array}{c}\text { Diagnoses: } \\
\text { "59010","59011","5902","59080","59081","5909","591","5933"," } \\
\text { 5934","5935","59381","59382","59589","5961","5962","5966","9 } \\
975 ", " 604 ", " 5991 ", " 7882 ", " 9963 ", " 5950 ", " 5970 ", \\
\text { Procedures: } \\
\text { "5501","5502","5503","5512","5593","5594","9761","9762","561" } \\
\text {,"5641","5673","5674","5675","5681","5682","5683","5684","568 } \\
\text { 5","5686","5689","5691","598" }\end{array}$ \\
\hline Gastrointestinal & $\begin{array}{c}\text { "5310","5311","5312","5313","5320","5321","5322","5323","540 } \\
\text { 0","5401","5409","5600","5601","5602","5603","5608","5609","7 } \\
\text { 876","9974",,5692", "5693","5695","5696","5793","00845" }\end{array}$ \\
\hline Neurologic & $\begin{array}{c}\text { "9970","99700","99701","99702","99709","436","951","952","95 } \\
\text { 3","954","955","956","3446","3530","354","355","7234" }\end{array}$ \\
\hline Infection & $\begin{array}{c}\text { "53641","51901","9985","993","038","0545","7907","99591","99 } \\
\text { 592" }\end{array}$ \\
\hline Vascular & $\begin{array}{c}\text { "4151","41511","41512","41519","451","4510","4511","4512","4 } \\
\text { 518","4519","4531","4534","45340","45341","45342","4538", } \\
\text { "4539","9972","9992","44422 ","44481","44489","433","4330", } \\
\text { "4331","4332","4333","4338","4339","434","4340","4341","4349" } \\
\text {,"436","437","4371","4372","4374","4373","4375","4376","4377", } \\
\text { "4378","4379","430","431","435","4599","4442","4448","9977" }\end{array}$ \\
\hline Wound infection & $\begin{array}{c}\text { "9983","99830","99831","99832","99833","9985","99859","9985 } \\
\text { 1","9986","567" }\end{array}$ \\
\hline Other & $\begin{array}{c}\text { "0418","2768","4589","584","7823","7824","7855","9950","9954" } \\
\text {,"9994","9996","9997","9984","9987","9988","9989","53640","53 } \\
\text { 642","53649","5793","99586" }\end{array}$ \\
\hline Transfusion & "9902","9904","9900", "9904", "9902", 9900" \\
\hline
\end{tabular}




\begin{tabular}{|c|c|c|c|c|}
\hline \multicolumn{5}{|c|}{$\begin{array}{l}\text { Supplementary Table 2. Multivariable logistic regression predicting delirium in } 3431 \\
632 \text { patients underwent one of ten major surgical oncology procedures, Nationwide } \\
\text { Inpatient Sample, 2003-2013 }\end{array}$} \\
\hline Predictors of delirium & OR & CI $2.50 \%$ & CI $97.50 \%$ & $\mathbf{p}$ \\
\hline Cystectomy & 9.25 & 7.73 & 11.07 & $<0.0001$ \\
\hline Pancreatectomy & 7.03 & 5.73 & 8.62 & $<0.0001$ \\
\hline Lung resection & 6.85 & 5.79 & 8.10 & $<0.0001$ \\
\hline Gastrectomy & 6.33 & 5.17 & 7.74 & $<0.0001$ \\
\hline Colectomy & 6.06 & 5.14 & 7.14 & $<0.0001$ \\
\hline Nephrectomy & 5.64 & 4.76 & 6.67 & $<0.0001$ \\
\hline Oophorectomy & 4.98 & 3.90 & 6.37 & $<0.0001$ \\
\hline Hysterectomy & 3.50 & 2.89 & 4.23 & $<0.0001$ \\
\hline Mastectomy & 1.15 & 0.91 & 1.45 & 0.2 \\
\hline Length of stay & 1.04 & 1.04 & 1.05 & $<0.0001$ \\
\hline Teaching hospital (ref. non-teaching) & 1.01 & 0.95 & 1.08 & 0.7 \\
\hline $\begin{array}{l}\text { Annual MSOP hospital volume low (ref. } \\
\text { high) }\end{array}$ & 1.00 & 0.93 & 1.08 & 0.9 \\
\hline Medium & 1.03 & 0.96 & 1.11 & 0.4 \\
\hline Hospital size small (ref. large) & 0.97 & 0.89 & 1.07 & 0.5 \\
\hline Medium & 0.96 & 0.89 & 1.02 & 0.2 \\
\hline 2009-2013 (ref. 2003-2008) & 1.43 & 1.35 & 1.52 & $<0.0001$ \\
\hline Age 55-64 (Ref. <55) & 1.90 & 1.67 & 2.16 & $<0.0001$ \\
\hline Age $\geq 65$ & 3.80 & 3.32 & 4.35 & $<0.0001$ \\
\hline Male (Ref. female) & 1.38 & 1.30 & 1.46 & $<0.0001$ \\
\hline African American (Ref. Caucasian) & 0.82 & 0.73 & 0.91 & 0.0002 \\
\hline Non-Caucasian & 0.88 & 0.82 & 0.94 & 0.0001 \\
\hline Charlson 1 (Ref. Charlson 0) & 1.07 & 1.01 & 1.13 & 0.03 \\
\hline Charlson $\geq 2$ & 1.20 & 1.12 & 1.29 & $<0.0001$ \\
\hline Medicaid (Ref. private ins) & 1.21 & 1.03 & 1.42 & 0.02 \\
\hline Medicare & 1.58 & 1.44 & 1.73 & $<0.0001$ \\
\hline Other & 0.97 & 0.81 & 1.15 & 0.7 \\
\hline Midwest (Ref. South) & 1.27 & 1.18 & 1.36 & $<0.0001$ \\
\hline Northeast & 1.03 & 0.95 & 1.11 & 0.5 \\
\hline West & 1.11 & 1.03 & 1.21 & 0.008 \\
\hline Dementia & 24.07 & 18.77 & 30.88 & $<0.0001$ \\
\hline Alcohol induced mental disorder & 0.90 & 0.65 & 1.25 & 0.5 \\
\hline Mood disorder & 2.43 & 2.02 & 2.93 & $<0.0001$ \\
\hline Non-organic disorder & 1.23 & 0.90 & 1.68 & 0.2 \\
\hline Anxiety disorder & 1.53 & 1.39 & 1.69 & $<0.0001$ \\
\hline Alcohol dependence & 14.51 & 12.68 & 16.62 & $<0.0001$ \\
\hline Schizophrenia disorder & 0.92 & 0.61 & 1.37 & 0.7 \\
\hline Drug dependence syndrome & 1.70 & 1.09 & 2.67 & 0.02 \\
\hline
\end{tabular}




\begin{tabular}{|l|c|c|c|c|}
\hline Non-dependent drug use & 1.11 & 1.02 & 1.20 & 0.01 \\
\hline Drug-induced disorder & 4.81 & 3.65 & 6.33 & $<0.0001$ \\
\hline
\end{tabular}

CI: confidence interval; MSOP: major surgical oncology procedures; OR: odds ratio. 